\title{
AN ALTERNATIVE TO THE PLÜCKER RELATIONS
}

\author{
HERBERT A. ROBINSON ${ }^{1}$
}

\begin{abstract}
It is shown how to obtain a set of homogeneous, degree $m$ polynomials in $\left(\begin{array}{l}n \\ m\end{array}\right)$ indeterminates over a field $F$ so that the associated algebraic variety is the set of decomposable elements in the $m$ th Grassmann space over an $n$-dimensional vector space over $F$. The same techniques are used to produce an analogous result for the tensor product of $\boldsymbol{m}$ finite dimensional vector spaces.
\end{abstract}

Let $V$ be an $n$-dimensional vector space over a field $F$, and denote the $m$ th Grassmann space over $V$ by $\bigwedge^{m} V$. (We assume throughout that $m \geqslant 2$.) It is well known that the set $\mathscr{D}=\left\{v_{1} \wedge \cdots \wedge v_{m}: v_{i} \in V, i=1, \ldots, m\right\}$ of decomposable elements in $\bigwedge^{m} V$ may be described as the algebraic variety associated with the set of so-called Plücker relations [2], [4]. An analogous result for the tensor product $V_{1} \otimes \cdots \otimes V_{m}$ of finite-dimensional vector spaces $V_{1}, \ldots, V_{m}$ has recently been obtained by Grone [1]. In both cases, the polynomials were homogeneous, degree 2. This paper shows how to produce alternative sets of (homogeneous, degree $m$ ) polynomials which define the same two varieties. While no claim is made that the new polynomials can be used more easily than Grone's polynomials or the notoriously cumbersome Plücker polynomials, the present approach has certain advantages: (1) The same methods work for $\wedge^{m} V$ and $V_{1} \otimes \cdots \otimes$ $V_{m}$, (2) the results have coordinate-free translations, and (3) the proofs are brief, as well as elementary.

The tensor space. Let $V_{i}^{*}$ denote the space of linear functionals on $V_{i}$, and suppose $f_{i} \in V_{i}^{*}, i=1, \ldots, m$. The universal factorization property of " $\otimes$ " permits the definitions of unique linear transformations $c_{j}: V_{1} \otimes \cdots \otimes V_{m}$ $\rightarrow V_{j}, j=1, \ldots, m$, and $c: V_{1} \otimes \cdots \otimes V_{m} \rightarrow F$ satisfying

$$
\begin{aligned}
& c_{j}\left(v_{1} \otimes \cdots \otimes v_{m}\right)=\left(\prod_{\substack{i=1 \\
i \neq j}}^{m} f_{i}\left(v_{i}\right)\right] v_{j}, \\
& c\left(v_{1} \otimes \cdots \otimes v_{m}\right)=\prod_{i=1}^{m} f_{i}\left(v_{i}\right),
\end{aligned}
$$

Received by the editors November $18,1976$.

AMS (MOS) subject classifications (1970). Primary 14M15; Secondary 15A69.

Key words and phrases. Decomposable tensor, Grassmann space, Plücker relations, tensor rank. ${ }^{1}$ This work was done while the author was a National Academy of Sciences-National Research Council Postdoctoral Research Associate at the National Bureau of Standards.

C American Mathematical Society 1977 
for all $v_{i} \in V_{i}, i=1, \ldots, m$. (The dependence of $c_{1}, \ldots, c_{m}, c$ on $f_{1}, \ldots, f_{m}$ will not be explicitly denoted.)

TheOREM 1. A tensor $z \in V_{1} \otimes \cdots \otimes V_{m}$ is decomposable if and only if for every choice of $f_{i} \in V_{i}^{*}, i=1, \ldots, m$, we have

$$
c_{1}(z) \otimes \cdots \otimes c_{m}(z)=[c(z)]^{m-1} z
$$

where $c_{1}, \ldots, c_{m}$, c are defined by (1), (2).

Proof. Obvious from the definitions and the fact that if $z \neq 0$, then there exist $f_{i} \in V_{i}^{*}, i=1, \ldots, m$, such that $c(z) \neq 0$.

By choosing bases of the spaces $V_{1}, \ldots, V_{m}$, and by using the usual induced basis in $V_{1} \otimes \cdots \otimes V_{m}$ and the dual bases in $V_{1}^{*}, \ldots, V_{m}^{*}$, we can convert Theorem 1 into a set of polynomial conditions on the coordinates of $z$ which are equivalent to the decomposability of $z$.

Let $V_{i}$ have the basis $v_{i 1}, \ldots, v_{i n_{i}}$ and let $f_{i 1}, \ldots, f_{i n_{i}}$ be the dual basis of $V_{i}^{*}, i=1, \ldots, m$. The set

$$
\left\{v_{1 \alpha(1)} \otimes \cdots \otimes v_{m \alpha(m)}: 1 \leqslant \alpha(i) \leqslant n_{i} \text { for } i=1, \ldots, m\right\}
$$

is a basis of $V_{1} \otimes \cdots \otimes V_{m}$ [3] (notation: $v_{1 \alpha(1)} \otimes \cdots \otimes v_{m \alpha(m)}=v_{\alpha}^{\otimes}$, $\Gamma=\left\{\alpha: 1 \leqslant \alpha(i) \leqslant n_{i}\right.$ for $\left.\left.i=1, \ldots, m\right\}\right)$, so $z$ has an expression

$$
z=\sum_{\alpha \in \Gamma} a_{\alpha} v_{\alpha}^{\otimes}, \quad a_{\alpha} \in F .
$$

Now, if $f_{i} \in\left\{f_{i 1}, \ldots, f_{i i_{i}}\right\}, i=1, \ldots, m$, say $f_{i}=f_{i \beta(i)}, \beta \in \Gamma$, then $c(z)$ and $c_{j}(z), j=1, \ldots, m$, may be computed using (1), (2), (4), and the duality of the $v_{i j}$ and $f_{i j}$ bases:

$$
\begin{aligned}
c(z) & =a_{\beta}, \\
c_{j}(z) & =\sum_{t=1}^{n_{j}} a_{\beta_{j t}} v_{j t},
\end{aligned}
$$

where $\beta_{j t} \in \Gamma$ is defined by

$$
\beta_{j t}(i)=\left\{\begin{array}{l}
\beta(i) \quad \text { if } i \neq j, \\
t \quad \text { if } i=j,
\end{array}\right.
$$

i.e., $\beta_{j t}$ is the sequence obtained from $\beta$ by replacing $\beta(j)$ by $t$. Compute each side of (3) using (5) and (6); (3) holds if and only if the coefficients of $v_{\alpha}^{\otimes}$ are equal for all $\alpha \in \Gamma$, i.e., if and only if

$$
\prod_{i=1}^{m} a_{\beta_{i \alpha(i)}}-a_{\beta}^{m-1} a_{\alpha}=0 .
$$

Thus, (8) holds for all $\alpha, \beta \in \Gamma$ if and only if (3) holds for every selection of $f_{i} \in\left\{f_{i 1}, \ldots, f_{i n_{i}}\right\}, i=1, \ldots, m$, and by Theorem 1 , this is equivalent to the decomposability of $z$. We have proved

THEOREM 2. The tensor $z=\Sigma_{\alpha \in \Gamma} a_{\alpha} v_{\alpha}^{\otimes} \in V_{1} \otimes \cdots \otimes V_{m}$ is decomposable if and only if (8) holds for all $\alpha, \beta \in \Gamma$. 
(Compare with the main result in [1].)

The Grassmann space. Let $f_{i} \in V^{*}, i=1, \ldots, m$. The universal factorization property of " $\wedge$ " permits the definitions of unique linear transformations $c_{j}: \wedge^{m} V \rightarrow V, j=1, \ldots, m$, and $c: \wedge^{m} V \rightarrow F$ satisfying

$$
c_{j}\left(v_{1} \wedge \cdots \wedge v_{m}\right)
$$

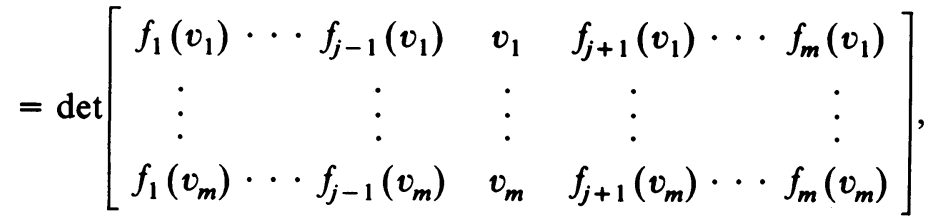

$$
\begin{aligned}
& c\left(v_{1} \wedge \cdots \wedge v_{m}\right)=\operatorname{det}\left[\begin{array}{ccc}
f_{1}\left(v_{1}\right) & \cdots & f_{m}\left(v_{1}\right) \\
\vdots & \vdots & \vdots \\
f_{1}\left(v_{m}\right) & \cdots & f_{m}\left(v_{m}\right)
\end{array}\right],
\end{aligned}
$$

where the right side of (9) is interpreted as a vector in $V$, e.g.,

$$
\begin{aligned}
& \operatorname{det}\left[\begin{array}{lll}
f_{1}\left(v_{1}\right) & v_{1} & f_{3}\left(v_{1}\right) \\
f_{1}\left(v_{2}\right) & v_{2} & f_{3}\left(v_{2}\right) \\
f_{1}\left(v_{3}\right) & v_{3} & f_{3}\left(v_{3}\right)
\end{array}\right]=-\operatorname{det}\left[\begin{array}{ll}
f_{1}\left(v_{2}\right) & f_{3}\left(v_{2}\right) \\
f_{1}\left(v_{3}\right) & f_{3}\left(v_{3}\right)
\end{array}\right] v_{1} \\
&+\operatorname{det}\left[\begin{array}{ll}
f_{1}\left(v_{1}\right) & f_{3}\left(v_{1}\right) \\
f_{1}\left(v_{3}\right) & f_{3}\left(v_{3}\right)
\end{array}\right] v_{2}-\operatorname{det}\left[\begin{array}{ll}
f_{1}\left(v_{1}\right) & f_{3}\left(v_{1}\right) \\
f_{1}\left(v_{2}\right) & f_{3}\left(v_{2}\right)
\end{array}\right] v_{3} .
\end{aligned}
$$

THEOREM 3. A skew-symmetric tensor $z \in \bigwedge^{m} V$ is decomposable if and only if for every choice of $f_{i} \in V^{*}, i=1, \ldots, m$, we have

$$
c_{1}(z) \wedge \cdots \wedge c_{m}(z)=[c(z)]^{m-1} z,
$$

where $c_{1}, \ldots, c_{m}$, c are defined by (9), (10).

Proof. The "if" part is obvious. For the converse, suppose $z=v_{1}$ $\wedge \cdots \wedge v_{m}$, and $f_{1}, \ldots, f_{m} \in V^{*}$ are given. Since each $c_{j}(z)$ is a linear combination of $v_{1}, \ldots, v_{m}, c_{1}(z) \wedge \cdots \wedge c_{m}(z)$ is a (possibly zero) multiple of $v_{1} \wedge \cdots \wedge v_{m}$, say

$$
c_{1}(z) \wedge \cdots \wedge c_{m}(z)=r z, \quad r \in F .
$$

From (9), it follows that

$$
f_{k}\left(c_{j}(z)\right)=\left\{\begin{array}{l}
c(z) \quad \text { if } k=j \\
0 \quad \text { if } k \neq j
\end{array}\right.
$$

so applying $c$ to both sides of (12) yields

$$
[c(z)]^{m}=r c(z), \quad c(z)\left(r-[c(z)]^{m-1}\right)=0 .
$$


Thus, (11) holds when $c(z) \neq 0$. If $c(z)=0$, then the adjugate of $\left[f_{j}\left(v_{i}\right)\right]$ is singular, and it follows easily that $c_{1}(z), \ldots, c_{m}(z)$ are linearly dependent, hence $c_{1}(z) \wedge \cdots \wedge c_{m}(z)=0$, so (11) still holds, and the proof is complete.

By introducing a basis of $V$, a dual basis of $V^{*}$, and the usual induced basis in $\wedge^{m} V$ [3], one may proceed in a manner analogous to that in the previous section, translating the criterion (11) into a set of $m$ th degree polynomial conditions on the coordinates of $z$ w.r.t. the induced basis. We choose to omit this slightly messy but straightforward calculation.

\section{REFERENCES}

1. Robert Grone, Decomposable tensors as a quadratic variety, Proc. Amer. Math. Soc. (to appear).

2. W. V. D. Hodge and D. Pedoe, Methods of algebraic geometry. I, Cambridge Univ. Press, London and New York, 1968.

3. Marvin Marcus, Finite dimensional multilinear algebra. I, Dekker, New York, 1973.

4. __ Finite dimensional multilinear algebra. II, Dekker, New York, 1975.

applied Mathematics Division, National Bureau of Standards, Washington, D. C. 20234

Current address: Department of Mathematics, Texas A \& M University, College Station, Texas 77843 